\title{
Time dependency of fluid flow near the top of the core
}

\author{
A. Bhattacharyya \\ Indian Institute of Geomagnetism, Colaba, Mumbai 400 005, India \\ (Received October 24, 1997; Revised June 26, 1998; Accepted July 16, 1998)
}

\begin{abstract}
Fluid flow in the core is assumed to consist of a slowly varying (on time scales $>$ magnetic diffusion time) part and a smaller, rapidly varying part as in the theory of the hydromagnetic dynamo put forward by Braginsky (1965). On the basis of this theory, geomagnetic secular variation models for the last 150 years are used to determine a rapidly varying, axisymmetric, poloidal motion of the fluid near the top of the core as a function of latitude in regions away from the equator. Approximations made in estimating this motion fail near the equator, thus restricting the estimates to latitudes $\geq 40^{\circ}$. Amplitude of the oscillating part of the axisymmetric poloidal flow is found to be $\leq 1 \mathrm{~km} / \mathrm{yr}$ in the northern hemisphere, and nearly $3 \mathrm{~km} / \mathrm{yr}$ in some parts of the southern hemisphere. The nature of temporal variation of this component differs significantly between the northern and southern hemispheres during the period under consideration.
\end{abstract}

\section{Introduction}

Time-dependent maps of the magnetic field at the coremantle boundary (CMB) have been constructed by Bloxham and Jackson (1992) (hereinafter BJ92) using most of the available data for the last three centuries, and assuming the mantle to be an insulator. The spatial variation of the magnetic field is described in terms of spherical harmonics in the usual way, while its temporal variation has been represented using a cubic B-spline basis. A secular variation model of the magnetic field at the CMB is thus available for the last 300 years.

Fluid flow in the outer core together with magnetic diffusion gives rise to temporal variation of the magnetic field at the CMB. Over time scales much shorter than the magnetic diffusion time associated with length scales $\geq$ few thousands of kilometers, the core may be considered to behave like a perfect conductor, such that the magnetic field lines are frozen in the fluid (Roberts and Scott, 1965). This argument has been used by a number of workers to estimate large scale fluid flow near the top of the core from secular variation models at the CMB (Whaler, 1980; Gubbins, 1982; Voorhies and Backus, 1985; Backus and Le Mouël, 1986; Lloyd and Gubbins, 1990; Bloxham and Jackson, 1991). Apart from the frozen-flux approximation the above estimates required some other assumption about the fluid flow in order to overcome the problem of non-uniqueness inherent in the determination of fluid flow near the top of the core from the induction equation (Backus, 1968). Hence the flow was considered to be either steady or geostrophic or toroidal in order to determine it uniquely from the frozen-flux induction equation.

In another approach (Rikitake, 1967; Honkura and Rikitake, 1972; Honkura and Matsushima, 1988; Matsushima and Honkura, 1989) to estimation of fluid flow in the outer core from a geomagnetic field model, it was as-

Copy right $($ C The Society of Geomagnetism and Earth, Planetary and Space Sciences (SGEPSS); The Seismological Society of Japan; The Volcanological Society of Japan; The Geodetic Society of Japan; The Japanese Society for Planetary Sciences. sumed that the non-axisymmetric poloidal magnetic field is maintained against Ohmic diffusion by the interaction between a strong zonal toroidal magnetic field and large-scale non-axisym-metric poloidal velocity fields as in an $\alpha \omega$-type geodynamo. The strong zonal toroidal field was considered to arise from the distortion of a large scale axisymmetric poloidal magnetic field by differential rotation, which is the so called $\omega$-effect. Rikitake (1967) and Honkura and Rikitake (1972) further assumed that non-zonal poloidal magnetic field of various modes and the toroidal magnetic field are in a steady state. These authors considered the representation of the non-zonal field in terms of standing and drifting parts suggested by Yukutake and Tachinaka (1969), and treated these two parts separately. Matsushima and Honkura (1988) expressed the scalar potential describing the observed poloidal field in terms of standing and drifting parts with periodically varying amplitudes, which was then used by Honkura and Matsushima (1988) to estimate fluid motion in the core at different epochs at 100 year intervals from 1600 to 2000, by the same method as Honkura and Rikitake (1972). The convection pattern was found to vary from one epoch to another. It was pointed out by Matsushima and Honkura (1992) that the $\omega$-effect may not be as strong as was assumed in the earlier papers, so that it is necessary to consider the other interaction terms also in the induction equation. Matsushima (1993) solved the induction equations for the toroidal and poloidal magnetic fields together with the Navier-Stokes equation for the toroidal velocity field to estimate fluid motion in the Earth's outer core from a geomagnetic field model. The author prescribed the radial dependence of the poloidal velocity field and minimized the temporal variations of the velocity and the magnetic fields. Thus at any particular epoch, the time derivative of the magnetic field at the CMB calculated using the velocity field derived by Matsushima (1993) was much smaller than given by the secular variation model for that epoch. Since secular variation of the poloidal field at the CMB contains important information about the geodynamo, 
it is necessary to consider it in attempts to estimate the temporal evolution of fluid flow in the outer core, which would shed light on the existence of lateral temperature variations at the base of the mantle.

A time-dependent geomagnetic field model was used by Benton and Celaya (1991) to determine a unique, unsteady surface flow at some chosen points on the CMB, under the frozen-flux approximation, by considering the temporal evolution of the fluid flow to be quartic. They found that at one illustrative point on the CMB, an exact fit to the 1900-1980 field model of Bloxham and Jackson (1989) required considerable evolution of the flow. Hulot et al. (1993) were the first to show clearly that core flows are highly time-dependent. This paper also gave the first account of a definite relationship of geomagnetic "jerks" with time-varying flow in the outer core. More recently, Jackson (1997) has obtained models of time-dependent core surface motions for the period 1840 1990 , using time-dependent maps of the magnetic field at the CMB (BJ92) and the frozen-flux and tangentially geostrophic flow approximations.

Fluid flow in the core is expected to show variations on short time scales because changes in the length of day (LOD) on decadal time scales are usually associated with changes of angular momentum of the core (Jault et al., 1988; Jackson et al., 1993). Further, Jackson (1997) has demonstrated in a forward calculation, that with steady motion of the fluid near the top of the core, it is not possible to fit the secular variation recorded at observatories to the required level of accuracy, even on short time scales of a few years. In the present paper, a method put forward by Bhattacharyya (1995), to use the secular variation of the axisymmetric poloidal magnetic field at the CMB to determine a rapidly oscillating (on time scales « magnetic diffusion time) part of the axisymmetric meridional flow near the top of the core, is utilized to study the temporal evolution of this component of surficial core flow over the period 1840-1990.

\section{Geodynamo Model}

A geodynamo model in which time variations of the magnetic field and fluid flow in the outer core are built in, is the hydromagnetic dynamo model of Braginsky (1965). In this model, the non-axisymmetric part $\mathbf{U}^{\prime}$ of the fluid flow in the core is considered to be a superposition of waves propagating in the $\phi$-direction, and the axially symmetric part of the flow is assumed to consist of a slowly varying $\left(\partial / \partial t \approx \eta c^{-2}\right.$, where $\eta$ is magnetic diffusivity and $c$ is the core radius) part and a rapidly oscillating part $\tilde{\mathbf{U}}$. This rapidly oscillating axisymmetric flow was termed oscillations by Braginsky (1965). The outer core fluid motion is thus expressed as:

$$
\mathbf{U}_{\text {tot }}=U \hat{\phi}+\mathbf{U}_{\mathrm{P}}+\tilde{\mathbf{U}}+\mathbf{U}^{\prime}
$$

where $U \hat{\phi}$ and $\mathbf{U}_{\mathrm{P}}$ are the toroidal and poloidal components of the slowly varying axisymmetric part of the flow, $\hat{\phi}$ being a unit vector in the azimuthal direction. Braginsky (1965) assumed that for a nearly axisymmetric dynamo, $\hat{U} \approx U^{\prime} \approx$ $U R_{\mathrm{m}}^{-1 / 2}$, where $R_{\mathrm{m}} \gg 1$ is the magnetic Reynolds number defined by $R_{\mathrm{m}}=U_{\mathrm{M}} c / \eta, U_{\mathrm{M}}$ being a typical value of $U$. It was also assumed in this model that the slowly varying axisymmetric part of the flow is dominated by the toroidal flow and $U_{\mathrm{P}} \approx U R_{\mathrm{m}}^{-1}$. With the assumption of large scale, slowly varying velocity and magnetic fields, Matsushima (1993) had found the toroidal velocity to be dominant at the CMB. In Braginsky's (1965) hydromagnetic dynamo the magnetic field was also represented in a manner similar to the velocity field:

$$
\mathbf{B}_{\mathrm{tot}}=B \hat{\phi}+\mathbf{B}_{\mathrm{P}}+\tilde{\mathbf{B}}+\mathbf{B}^{\prime} .
$$

The mantle is assumed to be an insulator such that at the top of the core, $B=0$ and $\tilde{B}_{\phi}=0$. Also the CMB is assumed to be a free-slip, spherical boundary, such that $U_{r}=\tilde{U}_{r}=U_{r}^{\prime}=0$ at $r=c$, where $U_{r}, \tilde{U}_{r}$, and $U_{r}^{\prime}$ are the radial components of $\mathbf{U}_{\mathrm{P}}, \tilde{\mathbf{U}}$ and $\mathbf{U}^{\prime}$ respectively.

Under these conditions, Braginsky (1965) found that for the time-varying axisymmetric poloidal field $\tilde{\mathbf{B}}_{\mathrm{P}}$, which can be expressed in terms of a vector potential $\tilde{A} \hat{\phi}$ :

$$
\tilde{\mathbf{B}}_{\mathrm{P}}=\nabla \times(\tilde{A} \hat{\phi}),
$$

only oscillations of the field of order $R_{\mathrm{m}}^{-3 / 2}$ can pass to the outside of the fluid core, and these are determined by

$$
\frac{\partial \tilde{A}}{\partial t}=\left[\tilde{\mathbf{U}}_{\mathrm{P}} \times \mathbf{B}_{\mathrm{P}}\right]_{\phi}
$$

Consideration of the momentum equations governing $\tilde{\mathbf{U}}$ and $\mathbf{U}_{\mathrm{P}}$ by Bhattacharyya (1995) showed that a term proportional to $\mathbf{U}_{\mathrm{P}}$ also makes a contribution to $\partial \tilde{\mathbf{B}}_{\mathrm{P}} / \partial t$ at the $\mathrm{CMB}$ of the same order, and hence must be included to obtain the following equation for its radial component:

$$
\frac{\partial \tilde{B}_{r}}{\partial t}=\frac{1}{c \sin \theta} \frac{\partial}{\partial \theta}\left(\tilde{U}_{z} B_{r}+U_{z} \tilde{B}_{r}\right)
$$

where the $z$-axis is along the Earth's rotation axis and $B_{r}$ is the radial component of the slowly varying, axisymmetric poloidal field $\mathbf{B}_{\mathrm{P}}$. Determination of fluid flow at the top of the core from the radial component of the classical frozen-flux induction equation suffers from the problem of non-uniqueness because it involves solving a single scalar equation for a two-dimensional vector flow. Equation (5) is derived from the longitudinally averaged radial component of the frozen-flux induction equation, and the problem is now reduced to solving this equation to determine a single scalar. Hence it is possible to obtain an unique solution as has been demonstrated by Bhattacharyya (1995).

The left-hand side of Eq. (5) may be expressed in terms of spherical harmonics:

$$
\frac{\partial \tilde{B}_{r}}{\partial t}=\sum_{n=1}^{N}(n+1)\left(\frac{a}{c}\right)^{n+2} \dot{g}_{n}^{0}(t) P_{n}(\cos \theta)
$$

where $a$ is the radius of the Earth, $P_{n}(\cos \theta)$ are Legendre polynomials, $\dot{g}_{n}^{0}(t)$ is the first derivative with respect to time of the Gauss coefficient $g_{n}^{0}(t)$, and $N$ is the truncation level of the spherical harmonic expansion for the geomagnetic secular variation model. The solution of Eq. (5) can be written as an expansion in Legendre polynomials (Bhattacharyya, 1995):

$$
\tilde{U}_{z} B_{r}+U_{z} \tilde{B}_{r}=\sum_{n=0}^{\infty} E_{n}(t) P_{n}(\cos \theta) .
$$




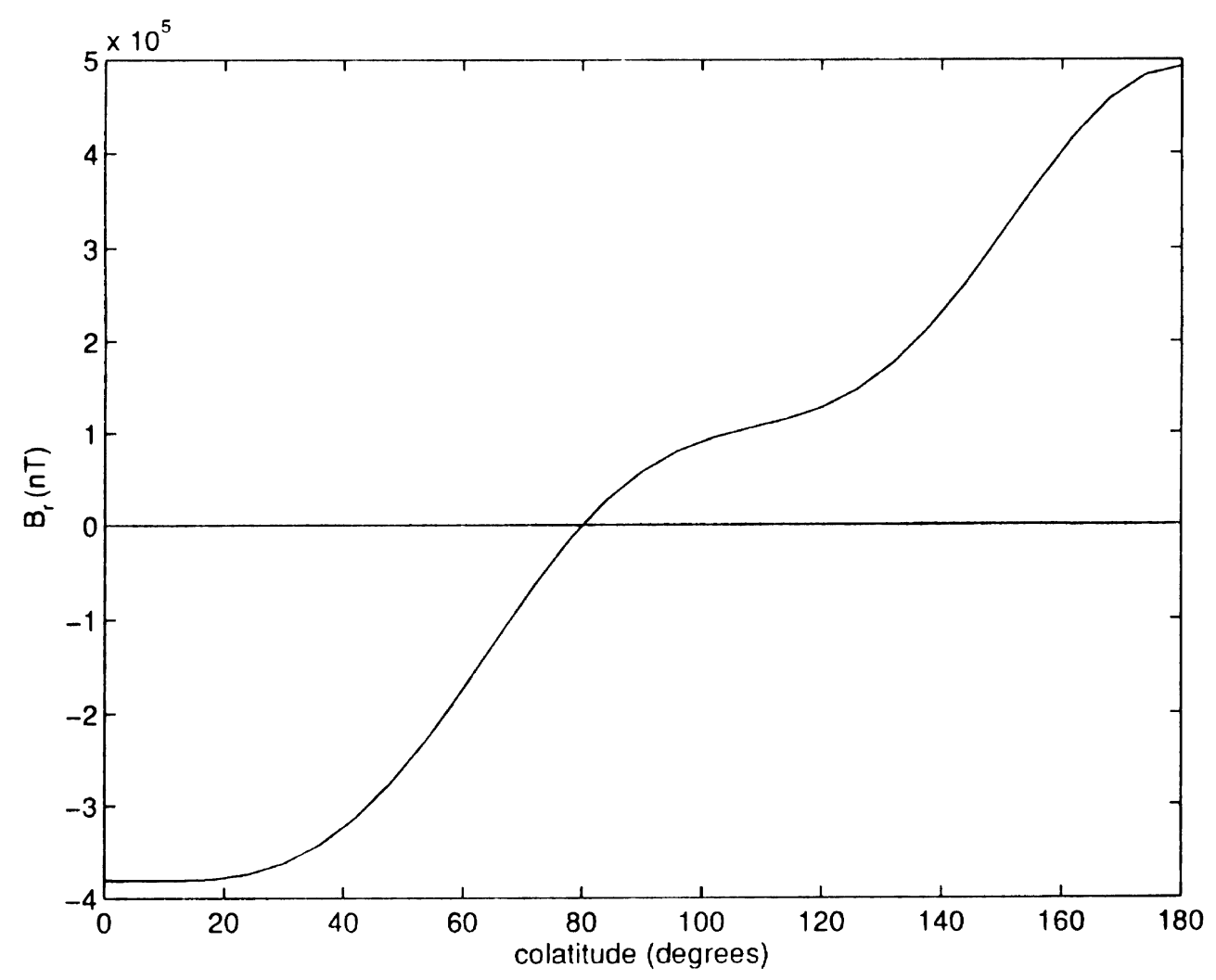

Fig. 1. Radial component $B_{r}$ of a steady, axisymmetric poloidal magnetic field at the CMB as a function of colatitude, derived from the time average normal-polarity palaeomagnetic field model of Kelly and Gubbins (1997).

The first term involving $E_{0}$ is independent of $\theta$ and is determined by the condition that on the CMB, at $\theta=0$ (or $\theta=\pi), \tilde{U}_{z}=\tilde{U}_{r}=0$ and $U_{z}=U_{r}=0$ :

$$
E_{0}=\frac{2}{3} c\left(\frac{a}{c}\right)^{3} \dot{g}_{1}^{0}
$$

For $n \neq 0, E_{n}$ is given by

$$
\begin{aligned}
E_{n}= & \frac{(n+2) c}{(2 n+3)}\left(\frac{a}{c}\right)^{n+3} \dot{g}_{n+1}^{0} \\
& -\frac{n c}{(2 n-1)}\left(\frac{a}{c}\right)^{n+1} \dot{g}_{n-1}^{0} .
\end{aligned}
$$

For all $n>N+1, E_{n}=0$ as a result of truncating the series expansion in Eq. (6) at $n=N$. The steady and fluctuating axisymmetric parts of the Navier-Stokes equation, under the Boussinesq and magnetostrophic approximations, were also used by Bhattacharyya (1995) to show that the contribution of the second term on the left hand side of Eq. (7) equals that of the first term. Thus the following solution is obtained for $\tilde{U}_{z}$ :

$$
\tilde{U}_{z}=\frac{1}{2} \sum_{n=0}^{N+1} E_{n}(t) P_{n}(\cos \theta) / B_{r} .
$$

\section{Fluctuations in Axisymmetric Poloidal Flow}

For the period 1840-1990, the Gauss coefficients for the expansion of the time rate-of-change of the geomagnetic field, in terms of spherical harmonics upto $N=14$, are available from the time-dependent models of the magnetic field at the CMB obtained in BJ92. These are used to compute the coefficients $E_{n}(t)$ for various epochs. The slowly varying component $B_{r}$ is, as such, an unknown in the calculation of $\tilde{U}_{z}$ from Eq. (10). However, it should be borne in mind that, at a given co-latitude $\theta$, by definition $B_{r}$ should not vary over a time-period of 150 years. Hence the short-period fluctuations in $\tilde{U}_{z}$ are determined entirely by the numerator of Eq. (10). Thus conclusions drawn from Eq. (10) regarding the pattern of short-period variations in the axisymmetric poloidal flow will not be affected by the choice of $B_{r}$ except in those regions where $B_{r}$ is so small as to invalidate the approximations that go into the derivation of Eq. (10) which involves neglect of higher order terms. This happens in the neighbourhood of the geographic equator where the radial component, $B_{r}$, of the axisymmetric part of the geomagnetic field is small. For the estimation of $\tilde{U}_{z}$ from Eq. (10), $B_{r}$ has been calculated from the axisymmetric part of the timeaverage palaeomagnetic field obtained by Kelly and Gubbins (1997). These authors used directional measurements from lavas, inclination measurements from ocean sediments and intensity measurements from lavas to arrive at geomagnetic field models for the past $5 \mathrm{Myr}$. The time-average normalpolarity palaeomagnetic field model obtained by Kelly and Gubbins is not axially symmetric, but the axisymmetric part is dominant. The radial component of the axisymmetric part which is used as $B_{r}$ in the present study is shown in Fig. 1. For reasons stated above, estimation of $\tilde{U}_{z}$ from Eq. (10) using this $B_{r}$ is restricted to latitudes $\geq 40^{\circ}$.

Another important parameter in the estimation of $\tilde{U}_{z}$ us- 


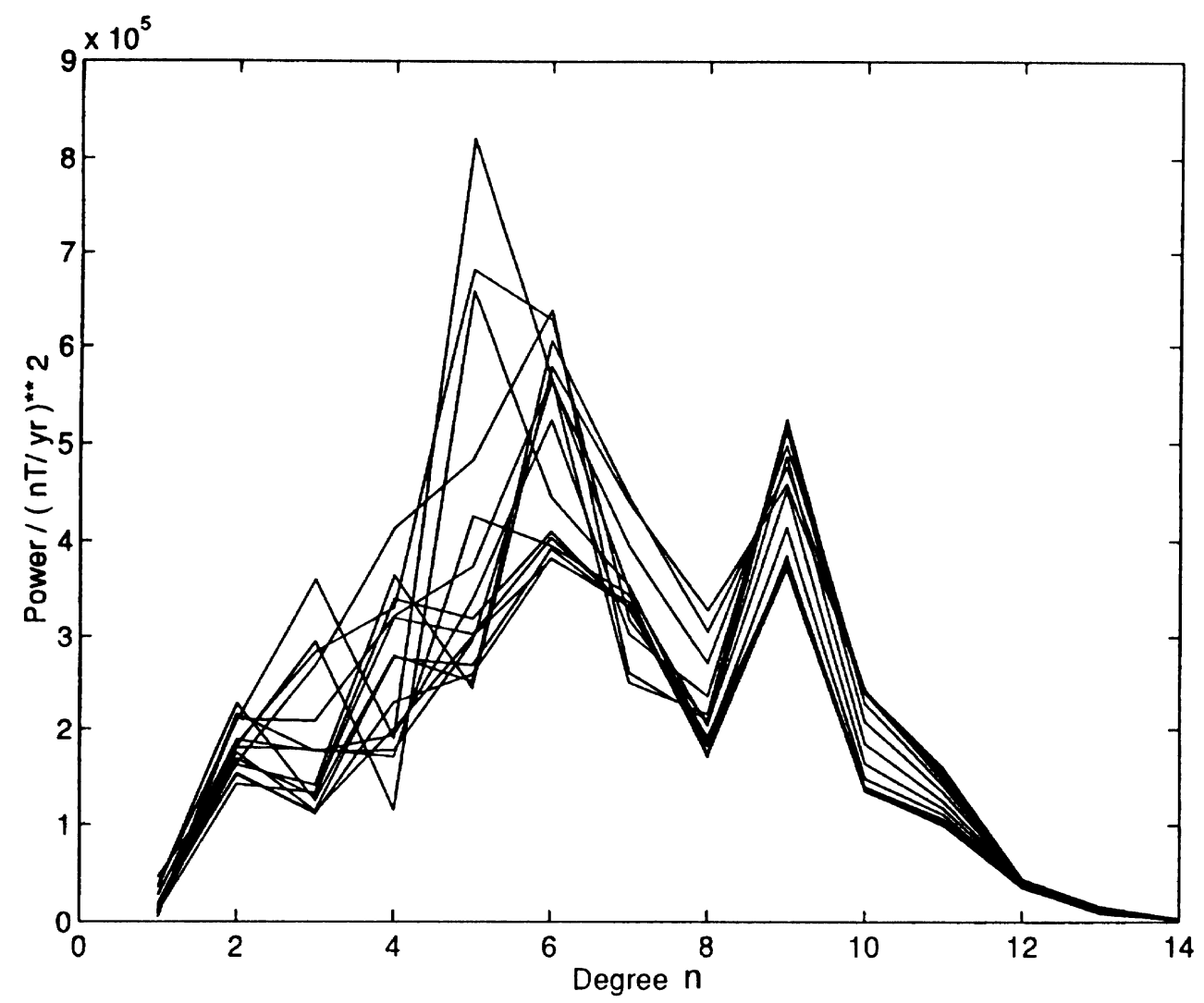

Fig. 2. Secular variation power at the CMB, calculated from the time-dependent geomagnetic field model BJ92 as a function of the harmonic degree $n$, for each decade of the period 1840-1990.

ing Eq. (10) is the truncation level $N$. The secular variation power at the $\mathrm{CMB}$, calculated from the time-dependent geomagnetic field model of BJ92 for each decade of the period 1840-1990, is plotted in Fig. 2 as a function of the harmonic degree $n$. This includes the non-axisymmetric part as well. An interesting feature that is revealed by this plot is the variability of the spectrum of the secular variation upto degree $n=6$, from one decade to another. It is seen that the peak in the power at $n=9$ emerges as a more stable feature during this period. This is mainly controlled by the regularising procedure used in obtaining the BJ92 model, which is based on the smoothest solutions compatible with the observations. This procedure involved minimization of two model norms, measuring roughness in the spatial and temporal domains respectively, and this is expected to result in greater stability for degrees higher than 7 or 8 . Secular variation of the geomagnetic field at the CMB has contributions from both fluid flow and magnetic diffusion, the latter contributing much more towards the temporal evolution of small scale features of the magnetic field than to the large scale features. Thus the temporal variability of the secular variation at $n \leq 6$ may be a direct manifestation of the temporal variability of fluid flow in the outer core. By this argument it may be sufficient to consider a truncation level $N=6$ for estimating the temporal evolution of fluid flow near the CMB. Nevertheless, for checking the convergence of the results, truncation levels of $N=8$ and $N=14$ have also been used in the calculations.

At the CMB, the meridional component $\tilde{U}_{\theta}$ of $\tilde{\mathbf{U}}_{\mathrm{P}}$ can be determined from $\tilde{U}_{z}$ because the radial component $\tilde{U}_{r}$ vanishes there, yielding

$$
\tilde{U}_{\theta}=-\tilde{U}_{z} / \sin \theta
$$

At the poles $\left(\theta=0^{\circ}, 180^{\circ}\right), \tilde{U}_{\theta}$ must be zero in order to be physically defined. This should be automatically satisfied by the solution obtained for $\tilde{U}_{\theta}$ from Eq. (11). Since $\tilde{U}_{z}$ vanishes at the poles according to the boundary conditions used in obtaining the solution Eq. (10), l'Hospital's rule has to be applied to Eq. (11) to demonstrate explicitly that $\tilde{U}_{\theta}$ also vanishes at the poles. Plots of $\tilde{U}_{\theta}$ as a function of $\theta$ for the epochs 1900 and 1960 are shown in Fig. 3 for $N=6,8$ and 14. It appears that increasing $N$ from 6 to 14 does not change the estimates drastically. A breakdown in the validity of the approximations leading to Eq. (10) explains the unphysically large values of $\tilde{U}_{\theta}$ in the neighbourhood of $B_{r}=0$, where there is a discontinuity in $\tilde{U}_{z}$ as given by Eq. (10). Retention of higher order terms would remove such a discontinuity in $\tilde{U}_{z}$ which is unphysical. Although $B_{r}$ becomes zero in the vicinity of $\theta=80^{\circ}$, it is seen from Fig. 1 that $B_{r}$ as a function of $\theta$ has a point of inflection in the neighbourhood of $120^{\circ}$, which has no counterpart in the northern hemisphere. Only for values of $\theta \geq 130^{\circ}$, the pattern of variation of $B_{r}$ with $\theta$ in the southern hemisphere is similar to the corresponding variation in the northern hemisphere. Also in this region $B_{r}$ is sufficiently large to make the right hand side of Eq. (4) the most dominant term in the expansion used by Braginsky (1965). Hence $\theta=130^{\circ}$ is chosen as one of the bounds. 

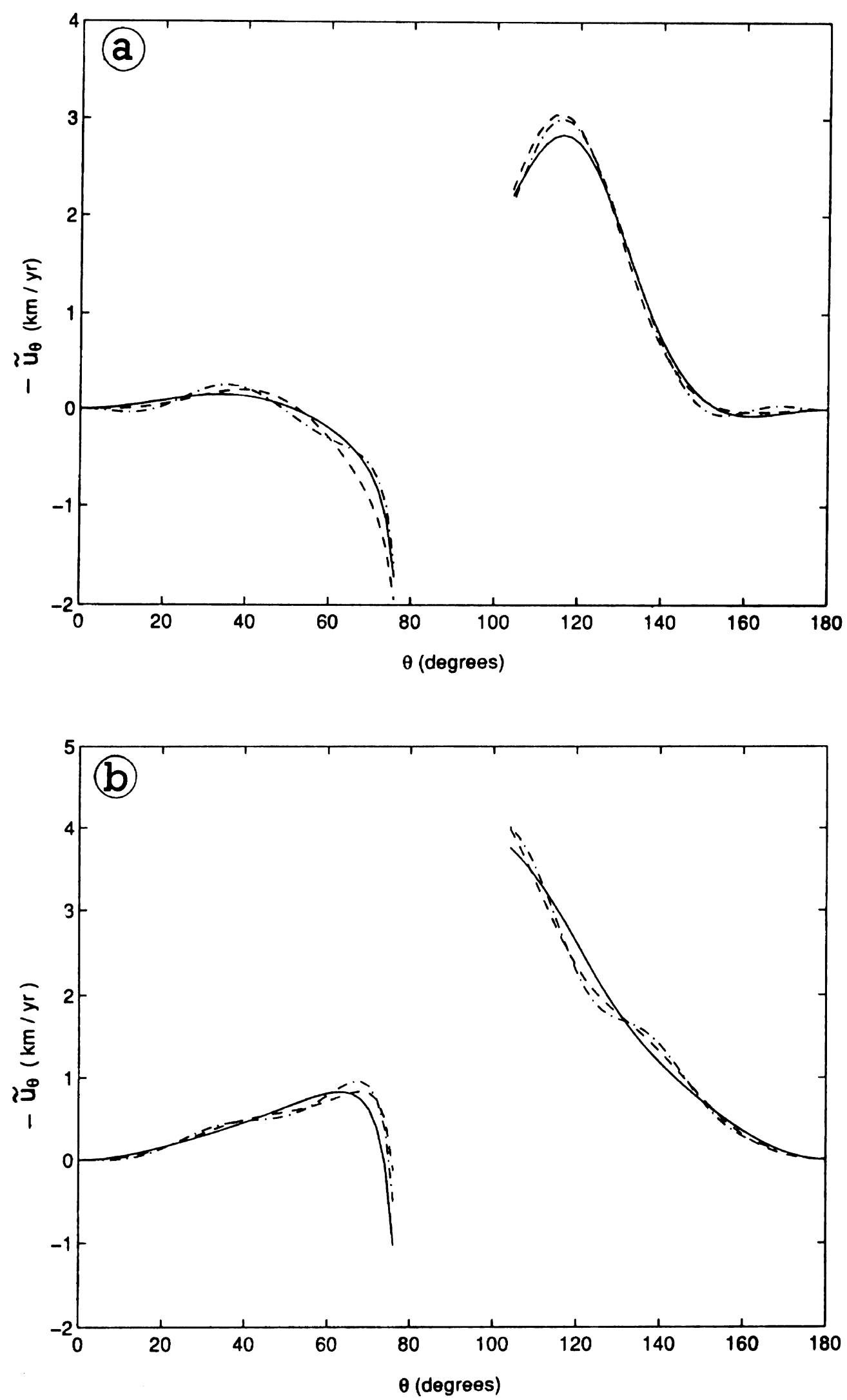

Fig. 3. Meridional component of the time-dependent part of the axisymmetric poloidal fluid flow as a function of colatitude for the epochs (a) 1900 and (b) 1960 computed from the secular variation model of BJ92. The truncation level used in the calculation are $N=6$ (solid line), $N=8$ (dash-dotted line) and $N=14$ (dashed line). 

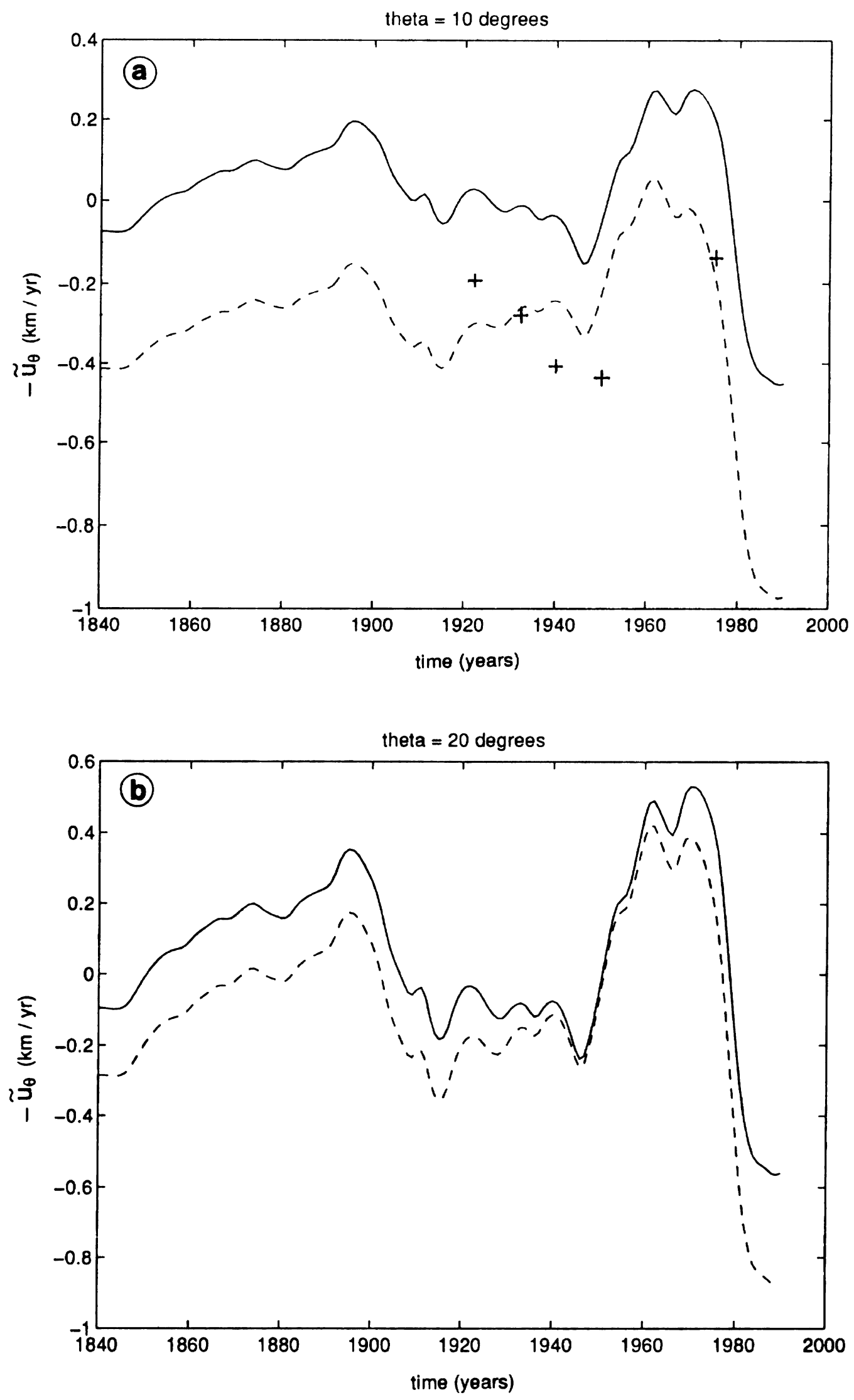

Fig. 4. Meridional component of the time-dependent part of the axisymmetric poloidal fluid flow as a function of time for selected latitudes (a-e) in the northern hemisphere. Solid and dashed lines are for $N=6$ and $N=14$ respectively. The crosses in (a) are computed using five different secular variation models for those particular epochs. 

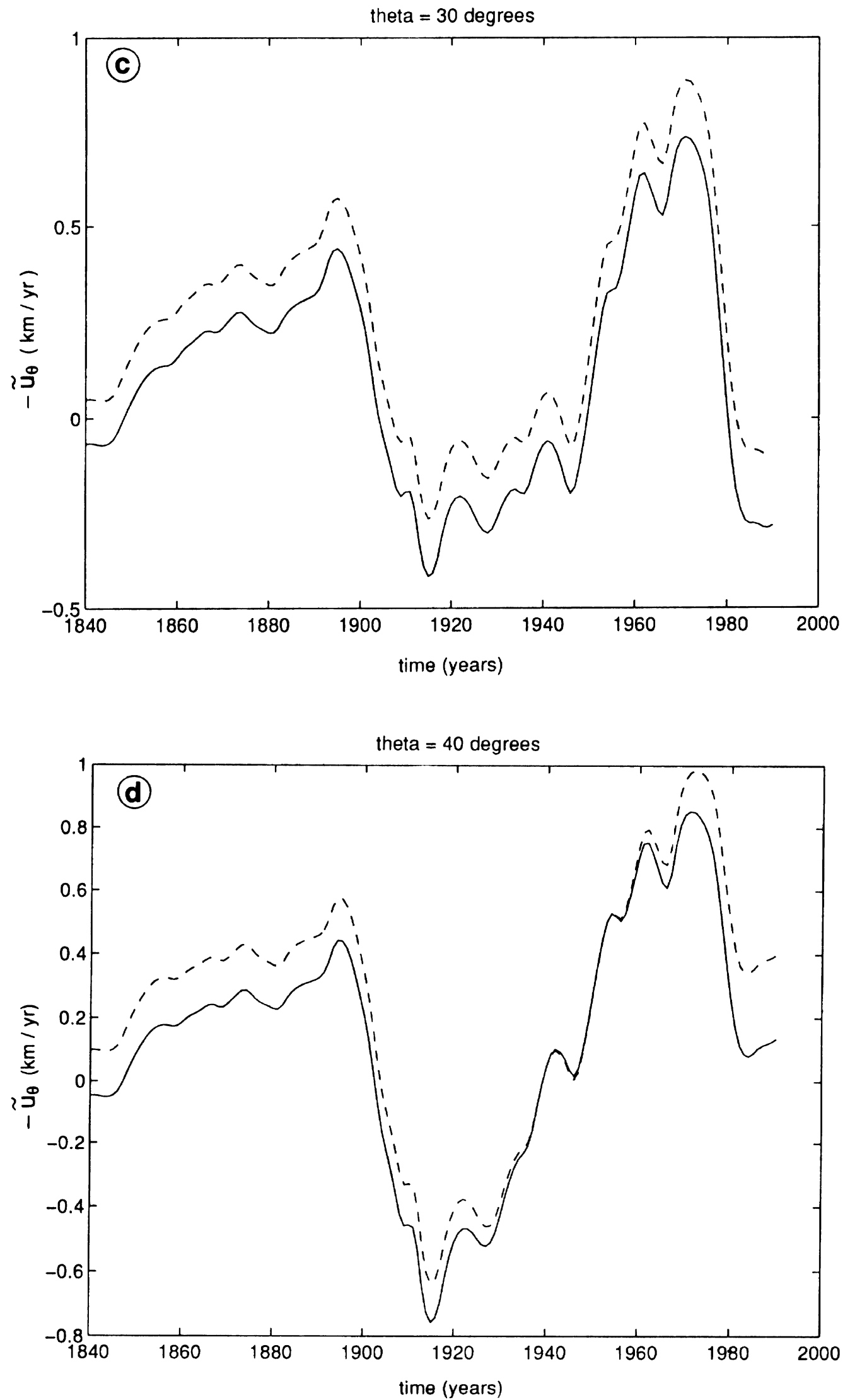

Fig. 4. (continued). 


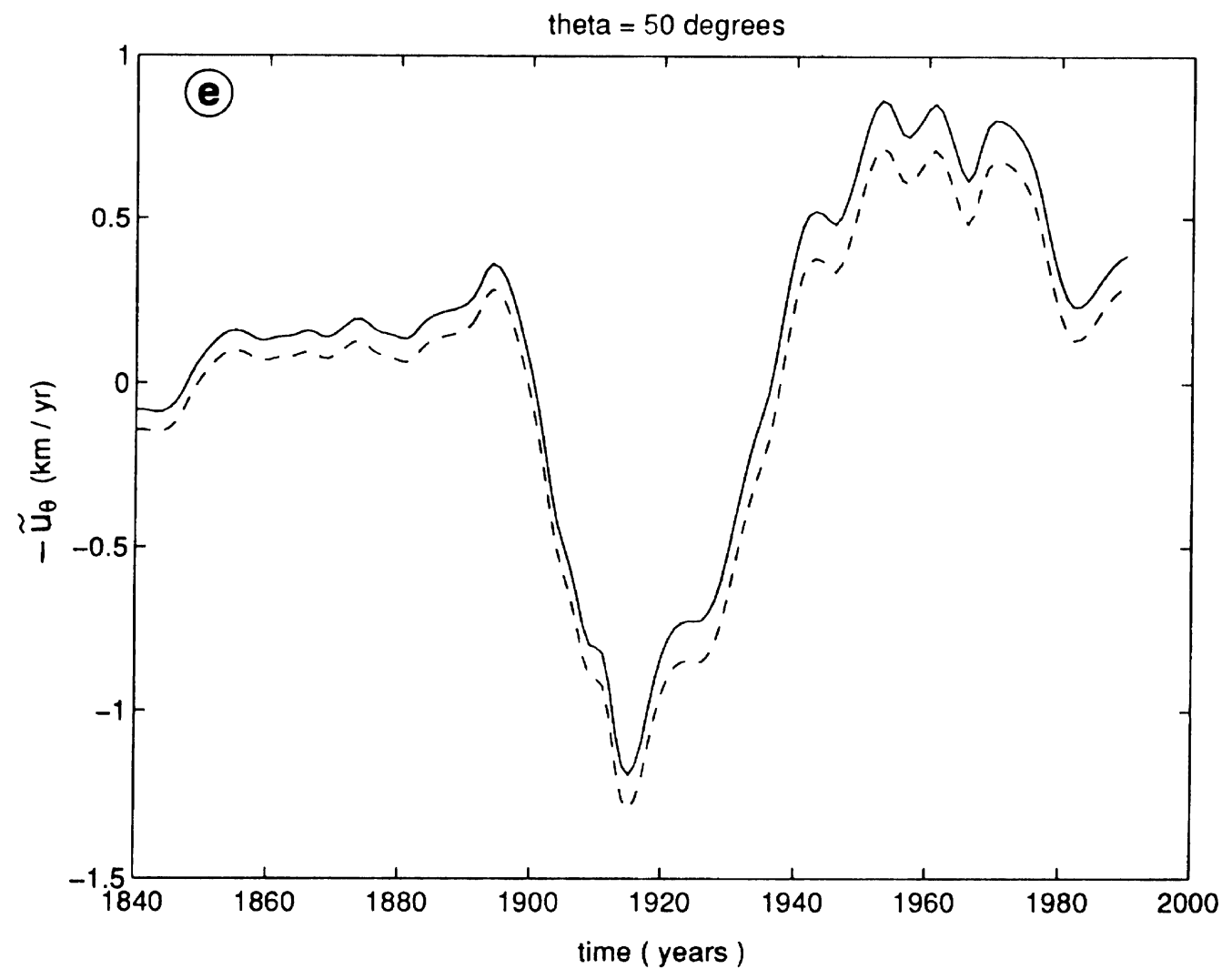

Fig. 4. (continued).

However, it can not be claimed that this is the correct bound. It is a non-controversial bound as far as a comparison between the two hemispheres is concerned. An estimate of the higher order corrections could yield a lower cutoff value of $\theta$ for which the computed values of $\tilde{U}_{\theta}$ may still be considered as reliable. In fact, a comparison of Figs. 3(a) and (b) shows more pronounced differences between epochs 1900 and 1960 for colatitudes below $\theta=130^{\circ}$, which can not be attributed to $B_{r}$. The choice of $\theta=50^{\circ}$ as the upper bound in the northern hemisphere is on the basis of Fig. 3(a), since for values of $\theta>60^{\circ}$ the estimates of $\tilde{U}_{\theta}$ appear to be affected by the singularity in the vicinity of $\theta=80^{\circ}$. Once again, a quantitative estimate of the cutoff has not been given, and the present choice may be an underestimate of the correct one. The main contention here is the difference between the patterns of temporal evolution of $\tilde{U}_{\theta}$ in the two hemispheres, which is not directly affected by the magnitude of $B_{r}$ at the higher latitudes. This is studied at selected latitudes for the 150 year period extending from 1840 to 1990 , using the timedependent secular variation model of BJ92. The results for northern and southern latitudes are shown in Figs. 4 and 5 respectively. Once again $\tilde{U}_{\theta}$ computed both with $N=6$ and $N=14$ are shown in these figures.

It will be recalled that the diffusion term itself was neglected in arriving at Eq. (4), as a consequence of the assumption that $R_{\mathrm{m}} \gg 1$. However in the estimated coefficients $\dot{g}_{n}^{m}$ for the secular variation of a time-dependent geomagnetic field model derived from observations, the contribution from diffusion is included. The smaller the length scale of the magnetic field, the more the magnetic field diffuses, hence there may be greater contributions from diffusion to the secular variation coefficients for higher degrees, and the temporal evolution of the secular variation coefficients $\dot{g}_{n}^{m}$ for $n>6$ could be such as to make the temporal variation of the computed $\tilde{U}_{\theta}$ significantly different for $N=6$ and $N=14$. However, it appears from Figs. 4 and 5 that, except at $\theta=170^{\circ}$, at the other colatitudes shown in these figures, temporal variation of the computed $\tilde{U}_{\theta}$ is not very different for $N=6$ and $N=14$. To check the sensitivity of the numerical method at different latitudes, five different models of secular variation listed in table 16 of Langel (1987) for epochs $1922.5,1932.5,1940,1950$ and 1975, are used to calculate $\tilde{U}_{\theta}$ at $\theta=10^{\circ}$ and $\theta=170^{\circ}$. These values are shown by crosses on Figs. 4(a) and 5(e) respectively. For epochs 1922.5 and 1932.5 the models have a truncation level of $N=6$ whereas for epochs 1940, 1950 and 1975 the models are truncated at $N=8$. Although the magnitude of $B_{r}$ at $\theta=170^{\circ}$ is greater than that at $\theta=10^{\circ}$, the results at $\theta=170^{\circ}$ are much more sensitive to the model used and hence do not appear to be reliable.

\section{Discussion}

The time-varying part, $\tilde{U}_{\theta}$, of the axisymmetric meridional flow determined here has to be added to the steady, axisymmetric part and the time-varying, non-axisymmetric part of the flow, which are unknown, in order to obtain a complete picture of the time-dependent fluid flow near the CMB. The purpose of studying only $\tilde{U}_{\theta}$ is simply to determine the time 

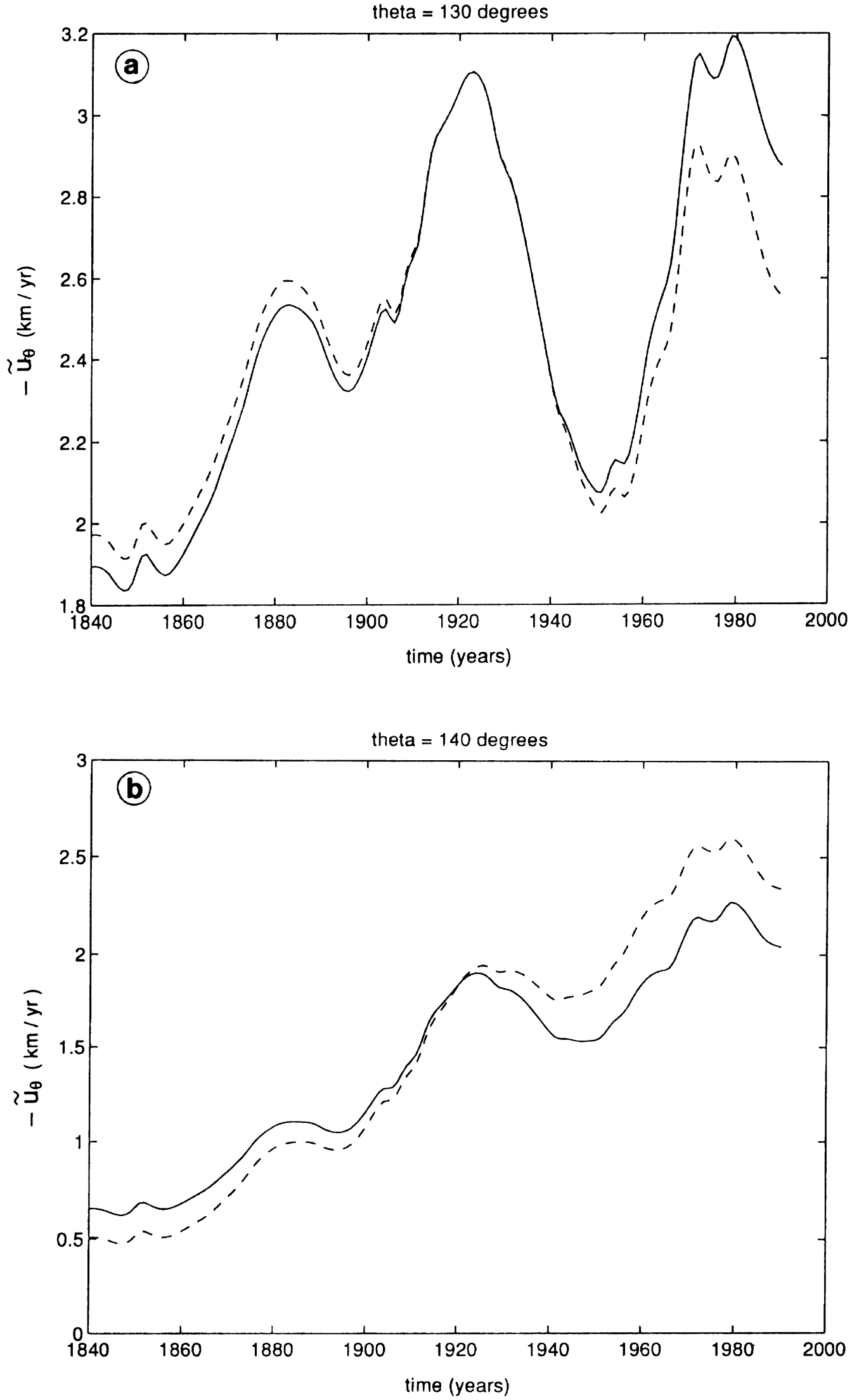

Fig. 5. Meridional component of the time-dependent part of the axisymmetric poloidal fluid flow as a function of time for selected latitudes (a-e) in the southern hemisphere. Solid and dashed lines are for $N=6$ and $N=14$ respectively. The crosses in (e) are computed using the same five secular variation models for those particular epochs, which were used to obtain the results marked by crosses in Fig. 4(a). 

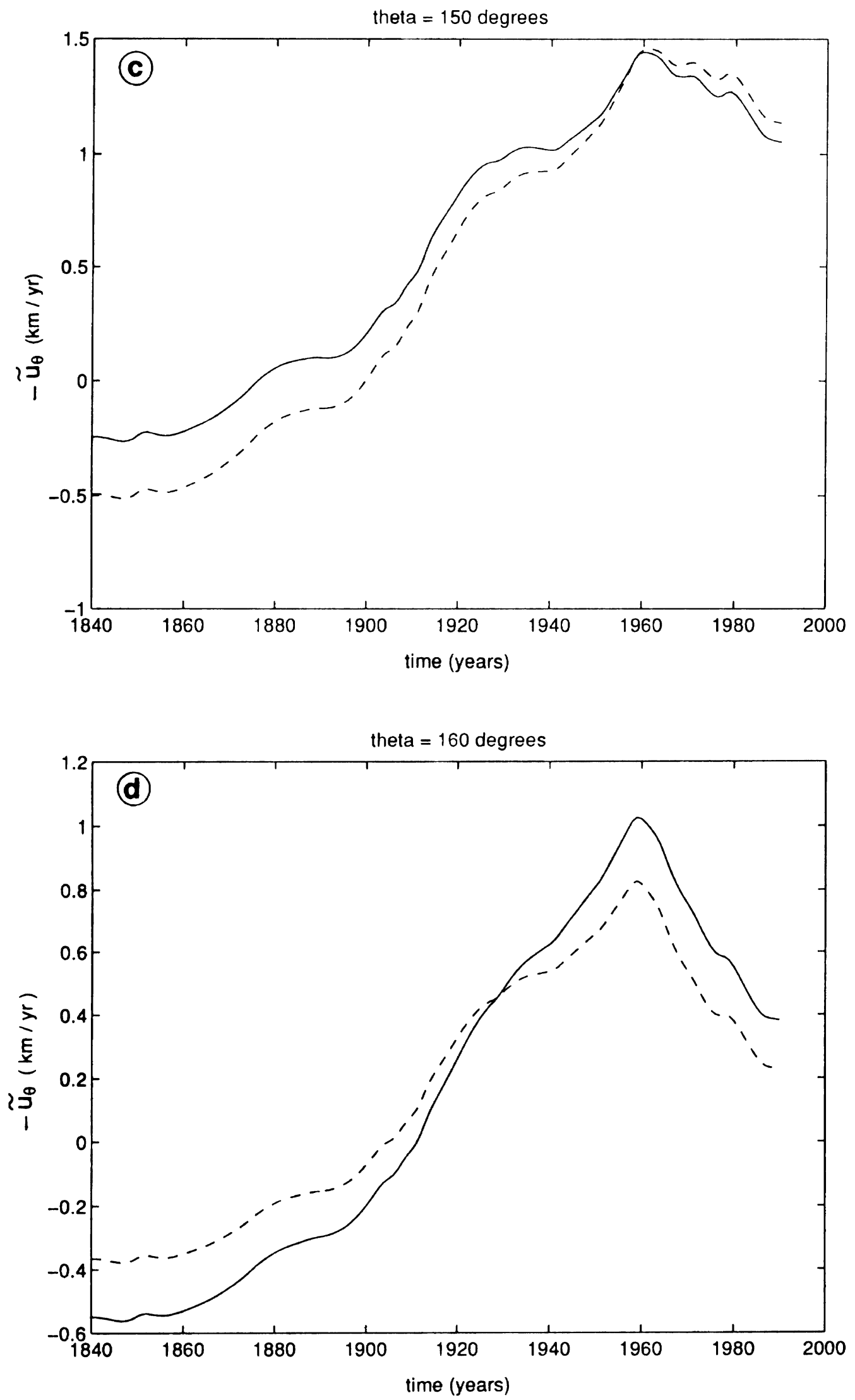

Fig. 5. (continued). 


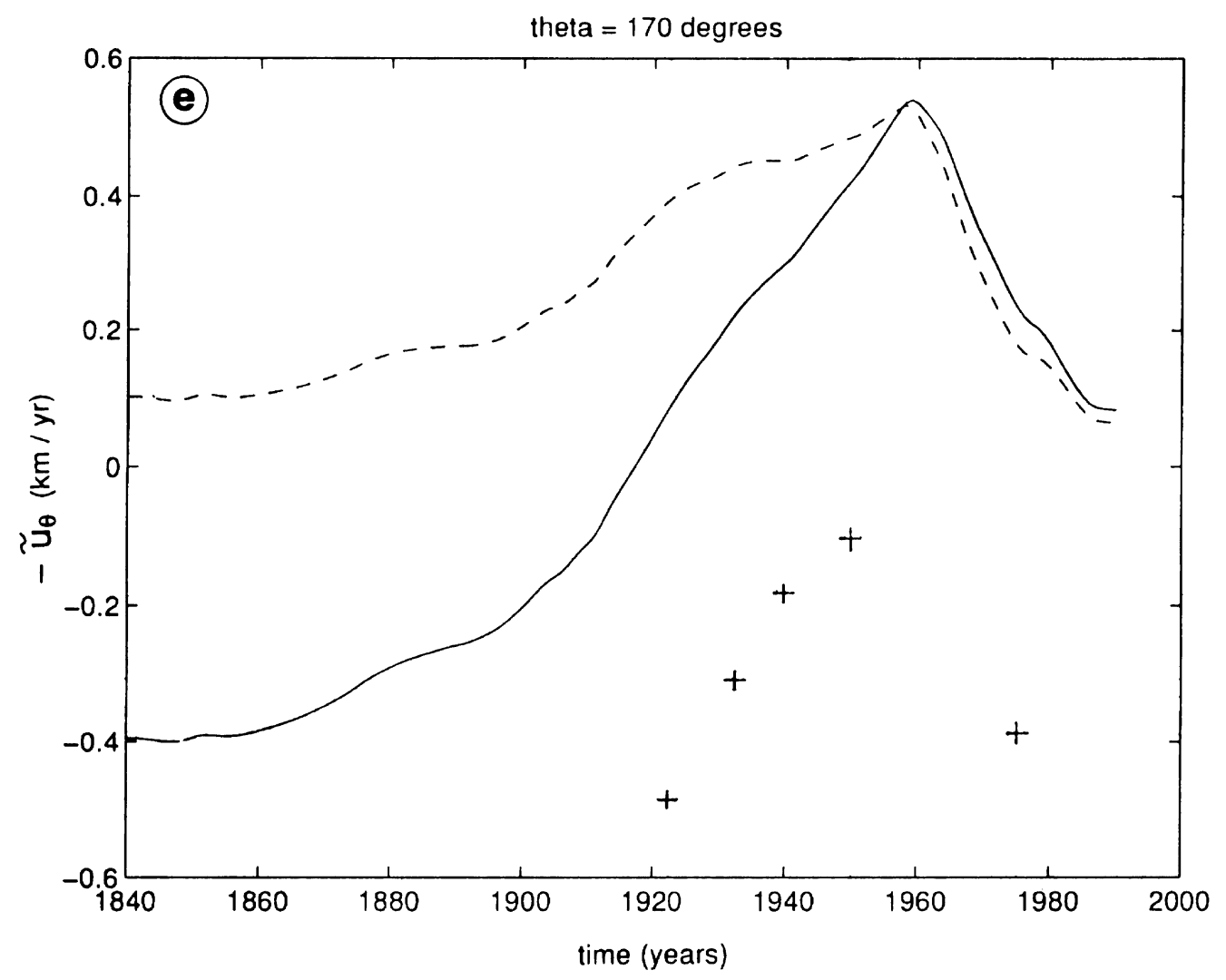

Fig. 5. (continued).

scales on which this fluid flow shows variations at different latitudes and to approximately estimate the magnitude of such fluctuations. In his theory of the hydromagnetic dynamo, Braginsky (I965) considered the non-axisymmetric part of the velocity to arise from a superposition of waves travelling in the $\phi$-direction. The basis for this was stated to be the observation that the geomagnetic field undergoes secular variations in which the non-axisymmetric components tend to drift predominantly towards the west, with different velocities. However, the axisymmetric part of the geomagnetic field also has a small component varying on "fast" time scales which are much shorter than the magnetic diffusion time $c^{2} / \eta$. Hence, Braginsky (1965) introduced the concept of "oscillations" which represent a rapidly oscillating component of the axially symmetric velocity that contains a slowly varying (on time scales $\geq c^{2} / \eta$ ) part as well. The poloidal part of this axisymmetric oscillatory motion may be written as

$$
\tilde{\mathbf{U}}_{\mathrm{P}}=\sum_{\mu} \mathbf{u}_{\mu} \exp ^{-i \phi_{\mu}}
$$

where the amplitudes $\mathbf{u}_{\mu}$ and the frequencies $\dot{\phi}_{\mu}$ are assumed to have a slow time variation, so that the phases $\phi_{\mu}$ of the oscillations are almost linear functions of time (Braginsky, 1965). An axisymmetric, poloidal flow would have only $r$ and $\theta$-components, of which the $r$-component vanishes at the top of the core. Hence $\tilde{U}_{\theta}$ defines the axisymmetric, oscillatory component of the poloidal flow and a study of its temporal evolution is expected to give an idea of the frequencies $\dot{\phi}_{\mu}$ involved. Estimates of $\tilde{U}_{\theta}$ in the present study, are used firstly to check the validity of the description provided by Eq. (12). Braginsky's (1965) theory requires that $\tilde{\mathbf{U}}_{\mathrm{P}}$ averaged over a period of time $>c^{2} / \eta$ should yield zero. Even for the short duration of 150 years studied here, a tendency towards such oscillatory behaviour, with periods less than 150 years, is seen at $\theta \leq 50^{\circ}$ in the northern hemisphere and $\theta \geq 150^{\circ}$ in the southern hemisphere, since at these latitudes $\tilde{U}_{\theta}$ changes sign in the course of these 150 years. However at $\theta=130^{\circ}$ and $\theta=140^{\circ}$ longer period oscillations also seem to be present which would require a longer data set to be studied. Nevertheless, even at these latitudes short period oscillations are seen, modulated by the longer period oscillations. With a sufficiently long time series, a Fourier analysis could be carried out to determine the frequencies, which is not feasible in the present study.

The amplitudes of the oscillations in the axisymmetric poloidal flow near the CMB determined here are uncertain to the extent of the uncertainity in $B_{r}$, which is model dependent. With the model of Kelly and Gubbins (1997) these amplitudes are found to be $\leq 1 \mathrm{~km} / \mathrm{yr}$ in the northern latitudes, while in the southern hemisphere, for $\theta=130^{\circ}$, this component of the fluid flow may be nearly $3 \mathrm{~km} / \mathrm{yr}$ at some epochs. As mentioned earlier, Hulot et al. (1993) had clearly shown that fluid flow in the outer core is highly time-dependent. A significant difference between the time-dependent fluid flow in the two hemispheres is also evident from the particle tracer plots shown in figure 3 of Jackson (1997). Voorhies (1993), on the other hand, had suggested on the basis of a simple calculation using decadal fluctuations in the length 
of day (LOD) that fluctuations in core surface flow is about $\pm 1 \mathrm{~km} / \mathrm{yr}$. This calculation was for a mean westward flow near the core surface.

Further differences in the temporal variation of fluid flow near the CMB in the two hemispheres is evident from Figs. 4 and 5. The axisymmetric meridional flow near the CMB shows greater variability on short time scales or "jerk"-like behaviour in the northern hemisphere than in the southern hemisphere. In the northern hemisphere for $20^{\circ} \leq \theta \leq 50^{\circ}$, there is rapid variation in this component of the flow close to 1895,1915 and 1970. Global rms value of secular variation in the radial component of the geomagnetic field at the earth's surface derived from model ufm1 of BJ92 displays the phenomena of "jerks" as can be seen in figure $1 \mathrm{~b}$ of Jackson (1997), whereas the global rms value of secular variation in the same component at the $\mathrm{CMB}$, derived from the same model, shows much smoother variation with time as in figure 1c of Jackson (1997). However, present results indicate that large scale fluid flow near the CMB shows more complex temporal behaviour than the geomagnetic field at least in the northern hemisphere. In the southern hemisphere, for $\theta \geq 150^{\circ}$, there is a sharp change in the axisymmetric meridional flow close to the 1960 epoch only. For $130^{\circ} \leq \theta \leq 140^{\circ}$, the temporal variation of this component of the flow is quite different showing oscillatory behaviour with a period of about 60 years superimposed on longer period variations.

In this paper, no attempt is made to estimate the time variation of the zonal toroidal flow. Hence the results obtained here cannot be directly compared with the root mean square velocity at the $\mathrm{CMB}$ or the zonal toroidal rms velocity over the CMB, estimated as a function of time by Jackson (1997) using the time-dependent field model ufm1 of BJ92. The flows derived in that work are the simplest in a spatial and temporal sense, while being compatible with the main field and secular variation values provided by the model ufm 1 of BJ92. Thus, all the short time scale variations seen in some of the fluid flows obtained in the present work are not expected to be seen in Jackson's (1997) results. Nevertheless, both the root mean square velocity and the zonal toroidal rms velocity at the CMB obtained by Jackson (1997) show sharp peaks near the 1915 epoch. The earlier paper by Hulot et al. (1993) has also discussed such peaks in the outer core fluid flow near the 1915 epoch. The present results have no contribution towards changes in LOD, and hence cannot be compared with LOD observations as such. It may simply be argued that whatever phenomenon causes rapid changes in the toroidal flow may also have similar repercussions on the poloidal flow. In that sense the present results for the northern hemisphere support the earlier results concerning time-dependent flows (Jault et al., 1988; Hulot et al., 1993; Jackson et al., 1993) and the recent findings of Jackson (1997). It should be noted that the particle tracer plots at the core surface presented in figure 3 of Jackson (1997), which show advection of a tracer during the period 1840-1990, clearly indicate that meridional components of the flows are mostly small compared to the zonal components. Hence the smallness of the timedependent part of the axisymmetric meridional flow derived here is in accord with Jackson's (1997) results.

The new result obtained here is the latitudinal variation in the time-dependency of the axisymmetric poloidal flow near the core surface. To start with, the fluid flow is assumed to be nearly steady and toroidal, as seen from Eq. (1), with a small time-varying component. The estimated time varying component is indeed small compared to earlier estimates of fluid flow at a particular epoch. The pattern of temporal evolution of fluid flow is however quite distinct in the two hemispheres. The time variation of the axisymmetric poloidal flow in the northern hemisphere obtained here has some similarities with that of the degree 1 zonal coefficient in a spherical harmonic representation of the flow (Jackson et al., 1993; Jackson, 1997). However, in the southern hemisphere for $\theta \geq 140^{\circ}$, the time variation is different being dominated by a sharp change around 1960. The uncertainities of the model ufm 1 (BJ92), which has been used in the present calculations, have not been considered in order to estimate the possible errors in the results obtained here. However, conclusions drawn about the gross features of temporal variation of the estimated fluid flow are expected to be unaffected by any such consideration, as in earlier calculations by other authors.

The assumption made in the present paper, about fluid flow in the outer core, as described by Eq. (1), is not incompatible with a mainly tangentially geostrophic flow near the core surface, since the largest component of the axisymmetric flow is assumed to be steady and toroidal, and a tangentially geostrophic, toroidal flow is purely zonal (Bloxham, 1990). According to equation (17) of Bhattacharyya (1995), the leading order contribution to the oscillatory component of the axisymmetric poloidal flow at the top of the core arises from the Lorentz force acting there, which depends mainly on the radial gradient of the steady part of the axisymmetric toroidal magnetic field near the CMB. Using the present method, it has been possible to estimate the time-varying, axisymmetric poloidal part of the flow near the $\mathrm{CMB}$, and this is found to be small compared to earlier estimates of fluid flow near the CMB obtained under the tangentially geostrophic assumption. This finding does not go against the hypothesis that the flow near the core surface is mainly tangentially geostrophic, with an additional small, oscillatory component arising from the Lorentz forces acting on the fluid there.

In conclusion, the results obtained here show significantly different temporal evolution of the axisymmetric poloidal component of fluid flow near the CMB in the northern and southern hemispheres. This may be a manifestation of the way in which magnetic field strength and fluid flow behave in the two hemispheres in an inherently non-linear MHD dynamo operating in the outer core. For instance, the peak magnetic field strength oscillates between the northern and southern hemispheres in an intermediate $\alpha \omega$-dynamo model (Glatzmaier and Roberts, 1993). Inhomogeneous boundary conditions existing at the $\mathrm{CMB}$ can also give rise to different time evolutions of fluid flow near the CMB in the two hemispheres. It is, of course, not possible to reach a conclusion regarding the relative contributions of the two effects towards the hemispherical differences in the temporal evolution of fluid flow near the CMB, based on the present study alone.

Acknowledgments. The author is grateful to J. Bloxham for providing the time-dependent geomagnetic field model $\mathrm{BJ} 92$ and $\mathrm{A}$ 
Jackson for providing a preprint of his work. The author gratefully acknowledges the valuable comments of the referees, $\mathrm{M}$. Matsushima and G. Hulot, which helped in making improvements on the original version.

\section{References}

Backus, G. E., Kinematics of the geomagnetic secular variation in a perfectly conducting core, Phil. Trans. Roy. Soc. Lond. A, 263, 239-266, 1968.

Backus, G. E. and J. L. Le Mouël, The region on the core-mantle boundary where a geostrophic velocity field can be determined from frozen flux magnetic data, Geophys. J. R. Astron. Soc., 85, 617-628, 1986.

Benton, E. R. and M. A. Celaya, The simplest, unsteady surface flow of a frozen-flux core that exactly fits a geomagnetic field model, Geophys. Res. Lett., 18, 577-580, 1991.

Bhattacharyya, A., An estimate of the radial gradient of the toroidal magnetic field at the top of the Earth's core, Phys. Earth Planet. Inter., 90, 81-90, 1995.

Bloxham, J., On the consequences of strong stable stratification at the top of Earth's outer core, Geophys. Res. Lett., 17, 2081-2084, 1990.

Bloxham, J. and A. Jackson, Simultaneous stochastic inversion for geomagnetic main field and secular variation, 2. 1820-1980, J. Geophys. Res., 94, 15,753-15,769, 1989.

Bloxham, J. and A. Jackson, Fluid flow near the surface of Earth's outer core, Rev. Geophys., 29, 97-120, 1991.

Bloxham, J. and A. Jackson, Time-dependent mapping of the magnetic field at the core-mantle boundary, J. Geophys. Res., 97, 19,537-19,563, 1992.

Braginsky, S. I., Theory of the hydromagnetic dynamo, Sov. Phys. JETP, 20, 1462-1471, 1965.

Glatzmaier, G. A. and P. H. Roberts, Intermediate dynamo models, J. Geomag. Geoelectr., 45, 1605-1616, 1993.

Gubbins, D., Finding core motions from magnetic observations, Phil. Trans. Roy. Soc. Lond. A, 306, 249-256, 1982.

Honkura, Y. and M. Matsushima, Time-dependent pattern of core motion inferred from fluctuations of standing and drifting non-dipole fields, $J$. Geomag. Geoelectr., 40, 1511-1522, 1988.

Honkura, Y. and T. Rikitake, Core motion as inferred from drifting and standing non-dipole fields, J. Geomag. Geoelectr., 24, 223-230, 1972.

Hulot, G., M. Le Huy, and J. L. Le Mouël, Secousses (jerks) de la variation sulaire et mouvements dans le noyau terrestre, C. R. Acad. Sci. Paris, 317, 333-341, 1993.

Jackson, A., Time-dependency of tangentially-geostrophic core surface motions, Phys. Earth Planet. Inter., 103, 293, 1997.

Jackson, A., J. Bloxham, and D. Gubbins, Time-dependent flow at the core surface and conservation of angular momentum in the coupled core- mantle system, in Dynamics of Earth's Deep Interior and Earth Rotation, edited by J. L. Le Mouël, D. E. Smylie, and T. Herring, pp. 97-107, AGU Geophysical Monograph 72, IUGG Volume 12, 1993.

Jault, D., C. Gire, and J. L. Le Mouël, Westward drift, core motions and exchanges of angular momentum between core and mantle, Nature, 333, 353-356, 1988.

Kelly, P. and D. Gubbins, The geomagnetic field over the past 5 million years, Geophys. J. Int., 128, 315-330, 1997.

Langel, R. A., The main field, in Geomagnetism, 1, edited by J. A. Jacobs, pp. 249-512, Academic, London, 1987.

Lloyd, D. and D. Gubbins, Toroidal fluid motion at the top of Earth's core, Geophys. J. Int., 100, 455-467, 1990.

Matsushima, M., Fluid motion in the Earth's core derived from the geomagnetic field and its implication for the geodynamo, J. Geomag. Geoelectr. 45, 1481-1495, 1993.

Matsushima, M. and Y. Honkura, Fluctuation of the standing and drifting parts of the Earth's magnetic field, Geophys. J., 94, 35-50, 1988.

Matsushima, M. and Y. Honkura, Large scale fluid motion in the Earth's outer core estimated from non-dipole magnetic field data, J. Geomag. Geoelectr., 41, 963-1000, 1989.

Matsushima, M. and Y. Honkura, Reexamination of fluid motion in the Earth's core derived from geomagnetic field data-Is the $\omega$-effect really strong in the core?, J. Geomag. Geoelectr., 44, 521-553, 1992.

Rikitake, T., Non-dipole field and fluid motion in the Earth's core, J. Geomag. Geoelectr., 19, 129-142, 1967.

Roberts, P. H. and S. Scott, On the analysis of the secular variation, 1, A hydromagnetic constraint: Theory, J. Geomag. Geoelectr., 17, 137-151, 1965.

Voorhies, C. V., Geomagnetic estimates of steady surficial core flow and flux diffusion: Unexpected geodynamo experiments, in Dynamics of Earth 's Deep Interior and Earth Rotation, edited by J. L. Le Mouël, D. E. Smylie, and T. Herring, pp. 113-125, AGU Geophysical Monograph 72, IUGG Volume 12, 1993.

Voorhies, C. V. and G. E. Backus, Steady flows at the top of the core from geomagnetic field models: The steady motions theorem, Geophys. Astrophys. Fluid Dyn., 32, 163-173, 1985.

Whaler, K. A., Does the whole of the Earth's core convect?, Nature, 287, 528-530, 1980.

Yukutake, T. and H. Tachinaka, Separation of the earth's magnetic field into the drifting and the standing parts, Bull. Earthq. Res. Inst., Univ. Tokyo, 47, 65-97, 1969.

A. Bhattacharyya (e-mail: archana@iig.iigm.res.in) 